\title{
New Asymmetric Approach to Natural Pyrrolizidines: Synthesis of $(+)$-Amphorogynine- A, $(+)$-Amphorogynine-D, and (+)-Retronecine
}

\author{
Caroline Roche, Katarína Kadlecíková, Amaël Veyron, Philippe Delair,*
}

Christian Philouze, and Andrew E. Greene

Université Joseph Fourier de Grenoble, Chimie Recherche (LEDSS), 38041 Grenoble Cedex, France

David Flot ${ }^{\dagger}$ and Manfred Burghammer

European Synchrotron Radiation Facility (ID13 Microfocus Beamline), 38043 Grenoble Cedex, France

\section{Supporting Information}

\section{Table of Contents}

${ }^{1} \mathrm{H}$ NMR of Compound $12 \mathrm{a}$

page $\mathrm{S} 2$

${ }^{13} \mathrm{C}$ NMR of Compound 12a

page $\mathrm{S} 3$

${ }^{1} \mathrm{H}$ NMR of Compound $12 \mathrm{~b}$

page $\mathrm{S} 4$

${ }^{13} \mathrm{C}$ NMR of Compound $12 \mathrm{~b}$

page $\mathrm{S} 5$

${ }^{13} \mathrm{C}$ NMR of Compound 13

page S6

${ }^{13} \mathrm{C}$ NMR of Compound 14

${ }^{1} \mathrm{H}$ NMR of Compound 1a (protected)

page $\mathrm{S} 7$

${ }^{13} \mathrm{C}$ NMR of Compound 1a (protected)

page $S 8$

${ }^{1} \mathrm{H}$ NMR of Compound 1a, Amphorogynine-A

page $\mathrm{S} 9$

${ }^{13} \mathrm{C}$ NMR of Compound 1a, Amphorogynine-A

page $\mathrm{S} 10$

page $\mathrm{S} 11$

${ }^{1} \mathrm{H}$ NMR of Compound 1d, Amphorogynine-D

page $\mathrm{S} 12$

${ }^{13} \mathrm{C}$ NMR of Compound 1d, Amphorogynine-D

page S13

${ }^{1} \mathrm{H}$ NMR of Compound 1d, Amphorogynine-D, in $\mathrm{CD}_{3} \mathrm{OD}$

page $\mathrm{S} 14$

${ }^{1} \mathrm{H}$ NMR of Compound 1d, methyl ester hydrochloride

page $\mathrm{S} 15$

${ }^{13} \mathrm{C}$ NMR of Compound 1d, methyl ester hydrochloride

page $\mathrm{S} 16$

${ }^{1} \mathrm{H}$ NMR of Compound 1d, ethyl ester

${ }^{13} \mathrm{C}$ NMR of Compound $1 \mathrm{~d}$, ethyl ester

page $\mathrm{S} 17$

${ }^{1} \mathrm{H}$ NMR of Compound 20a

page $\mathrm{S} 18$

page $\mathrm{S} 19$

${ }^{13} \mathrm{C}$ NMR of Compound 20a

page $\mathrm{S} 20$

${ }^{1} \mathrm{H}$ NMR of Compound $20 \mathrm{~b}$

page $\mathrm{S} 21$

${ }^{13} \mathrm{C}$ NMR of Compound 20b

page $\mathrm{S} 22$

${ }^{1} \mathrm{H}$ NMR of Compound 21

page $\mathrm{S} 23$

${ }^{13} \mathrm{C}$ NMR of Compound 21

page S24

${ }^{1} \mathrm{H}$ NMR of Compound 2, Retronecine

page $\mathrm{S} 25$

${ }^{13} \mathrm{C}$ NMR of Compound 2, Retronecine

page $\mathbf{S} 26$ 


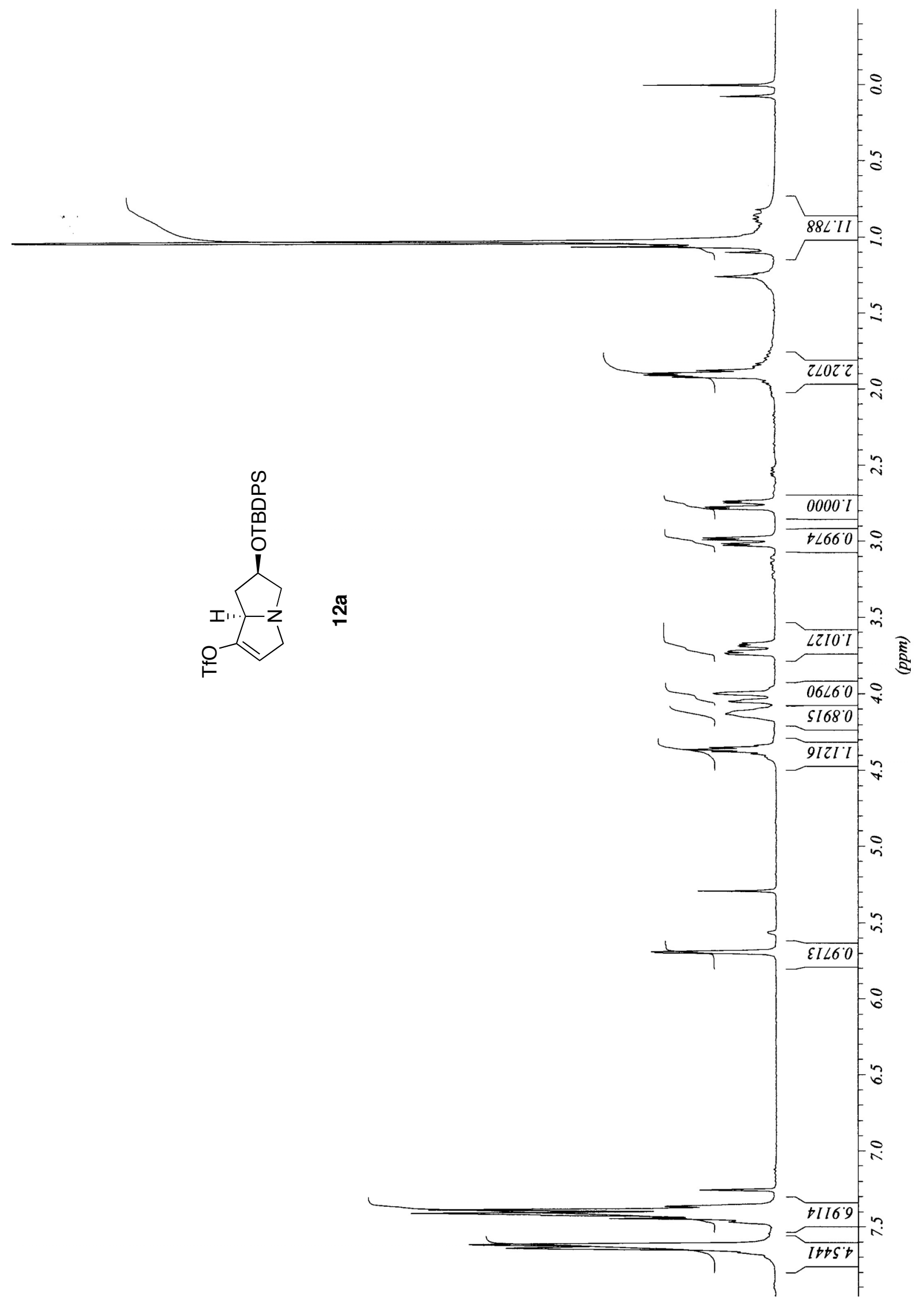




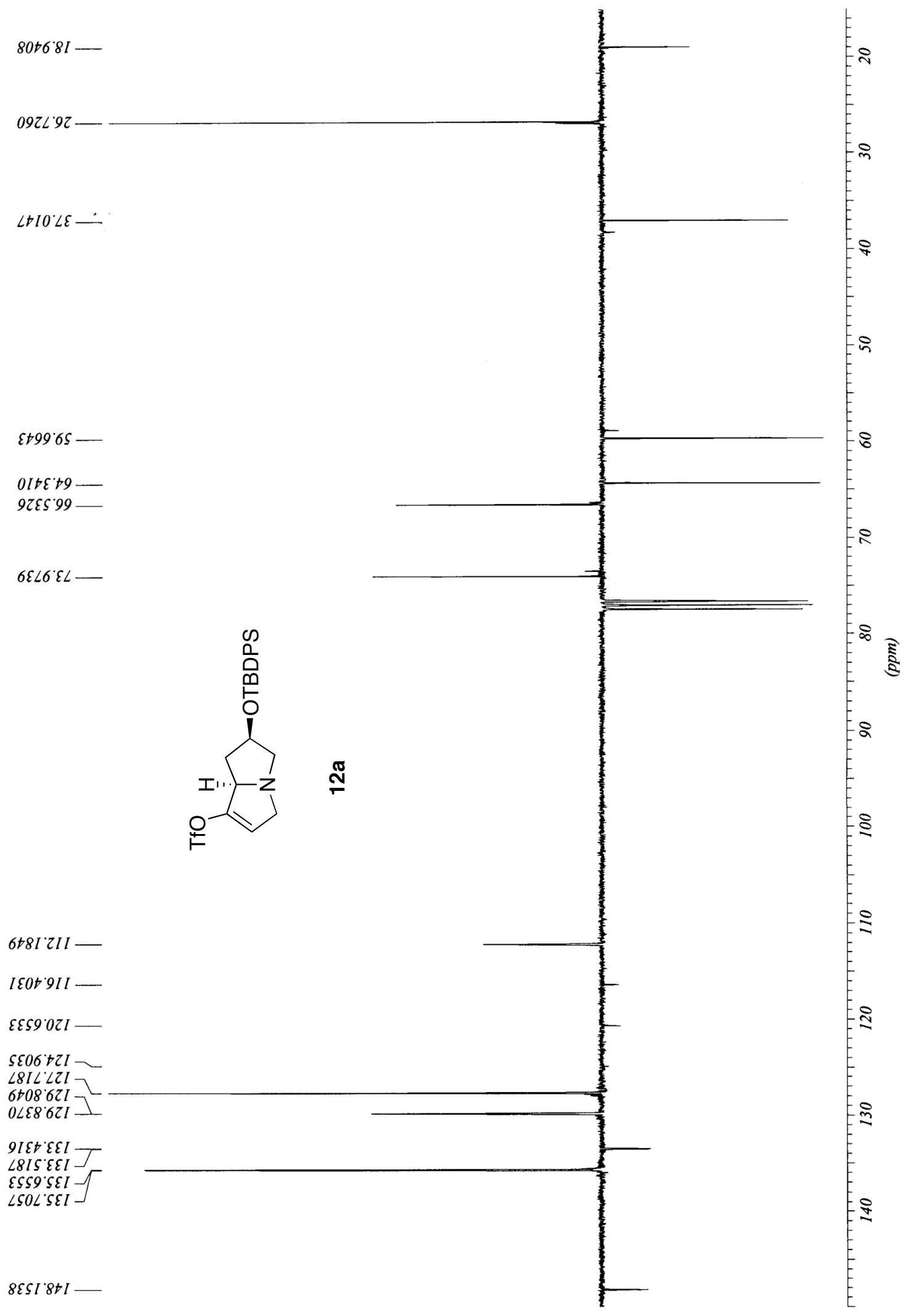




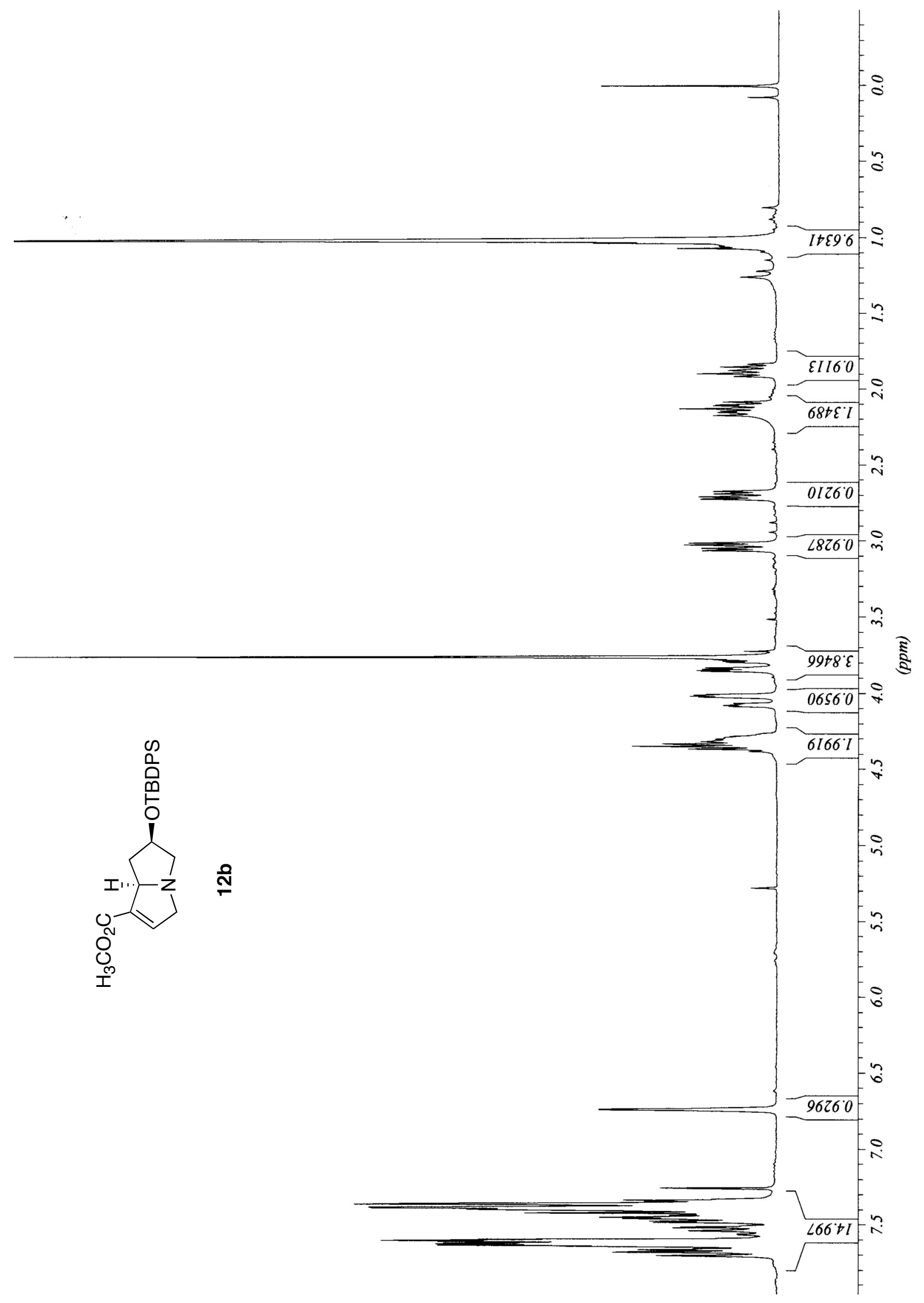


$S \angle L 68 I-$

$68+\angle 92-$

$s 26 I 6 \mathcal{E}$

ISZt IS

$0+\varepsilon S \cdot \varepsilon$ $8 \mathcal{B}+8^{\circ} \mathcal{E} \mathrm{C}$

$\varepsilon+9 I 69$

$6 I 50^{\circ}+L$

$\angle S 8 S^{\circ} \angle Z I$

$8 \angle 19^{\circ} \angle Z I$

$68 \angle E \cdot 8 Z I$

t6\&S $82 I$

$9 \varepsilon S 99^{\circ} 6 Z I-r$

9S00'ZEI -T

$\varepsilon \varepsilon+<\varepsilon \varepsilon I-F$

$S \angle 08^{\circ} \mathcal{E E} I$

SIt9 $S \mathcal{S E I}$

$6169^{\circ} \mathrm{SEI}$

$\angle 0 I 8^{\circ} 9 \mathcal{E} I$

OILO $6 E I$

$026 I+9 I-$

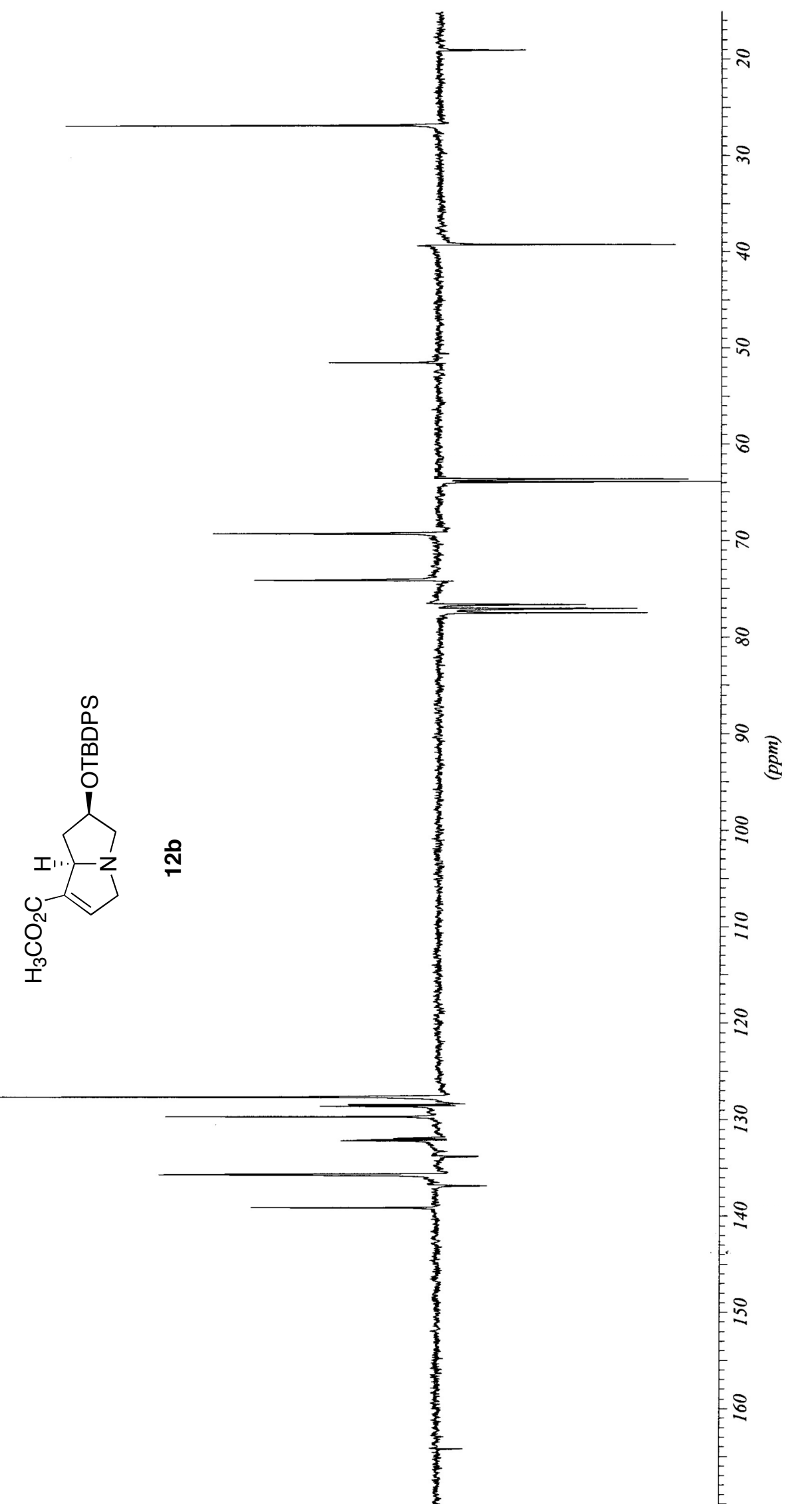


\&£206I -

$60 \varepsilon z \cdot 9 \tau$

$19 \varepsilon 8.92$

$0+9+\angle \varepsilon$

$6 z<t<t$

$018 S^{\circ} I S$

$\varepsilon+98^{\circ} \varepsilon \varsigma$

$\angle+26.19$

$\varepsilon 0+\angle \cdot E 9$

$z 6 \varepsilon 9^{\circ} \varepsilon L$

$S 66 S^{\circ} \angle Z I$

$S+S 9^{\circ} \angle Z I$

$\angle 26 \varepsilon 827$

ZESS 821

$59 \angle 90^{\circ} 621$

$692<62 I]$

$\varepsilon \angle \angle 8^{\circ} I \varepsilon I$

$+606^{\circ} I \varepsilon I$

$8+10 \cdot z \varepsilon I$

$8 \angle t I ' Z \varepsilon I$

$I \angle S \angle E E I$

9819 SEI

$869^{\circ}{ }^{\circ} \mathcal{E} I$

$\angle Z 9 \phi^{\circ} \varepsilon \angle I$

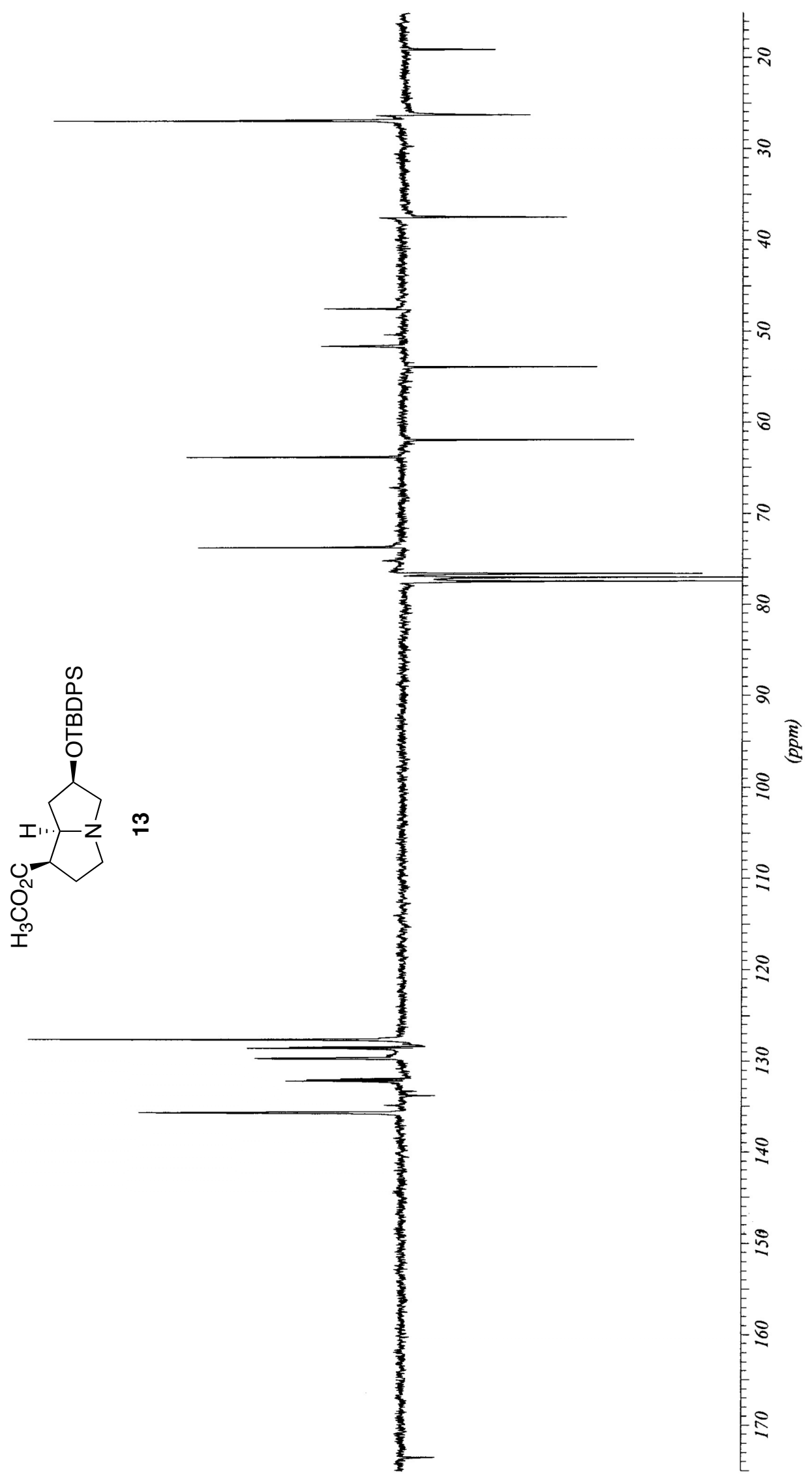




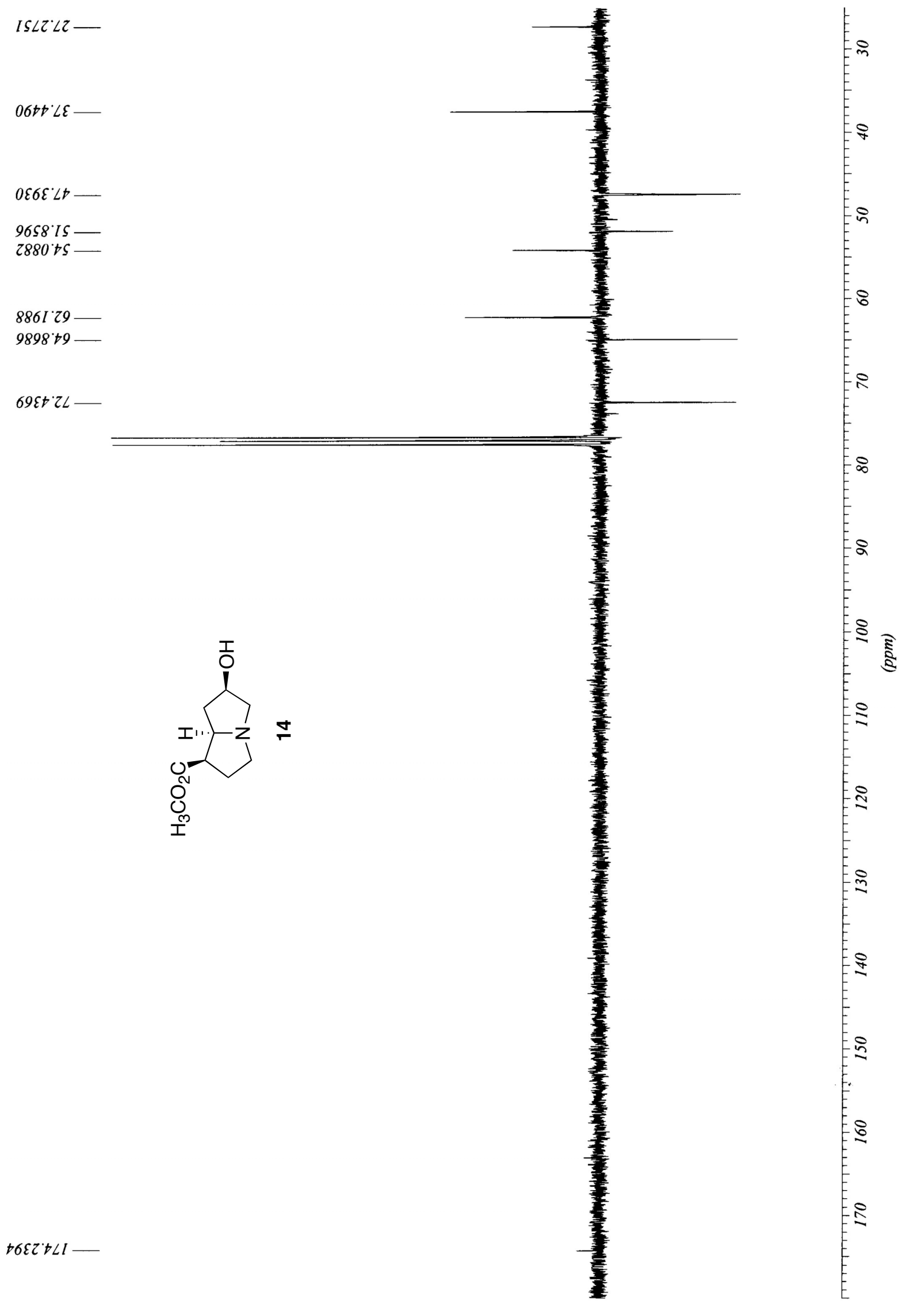

S7 


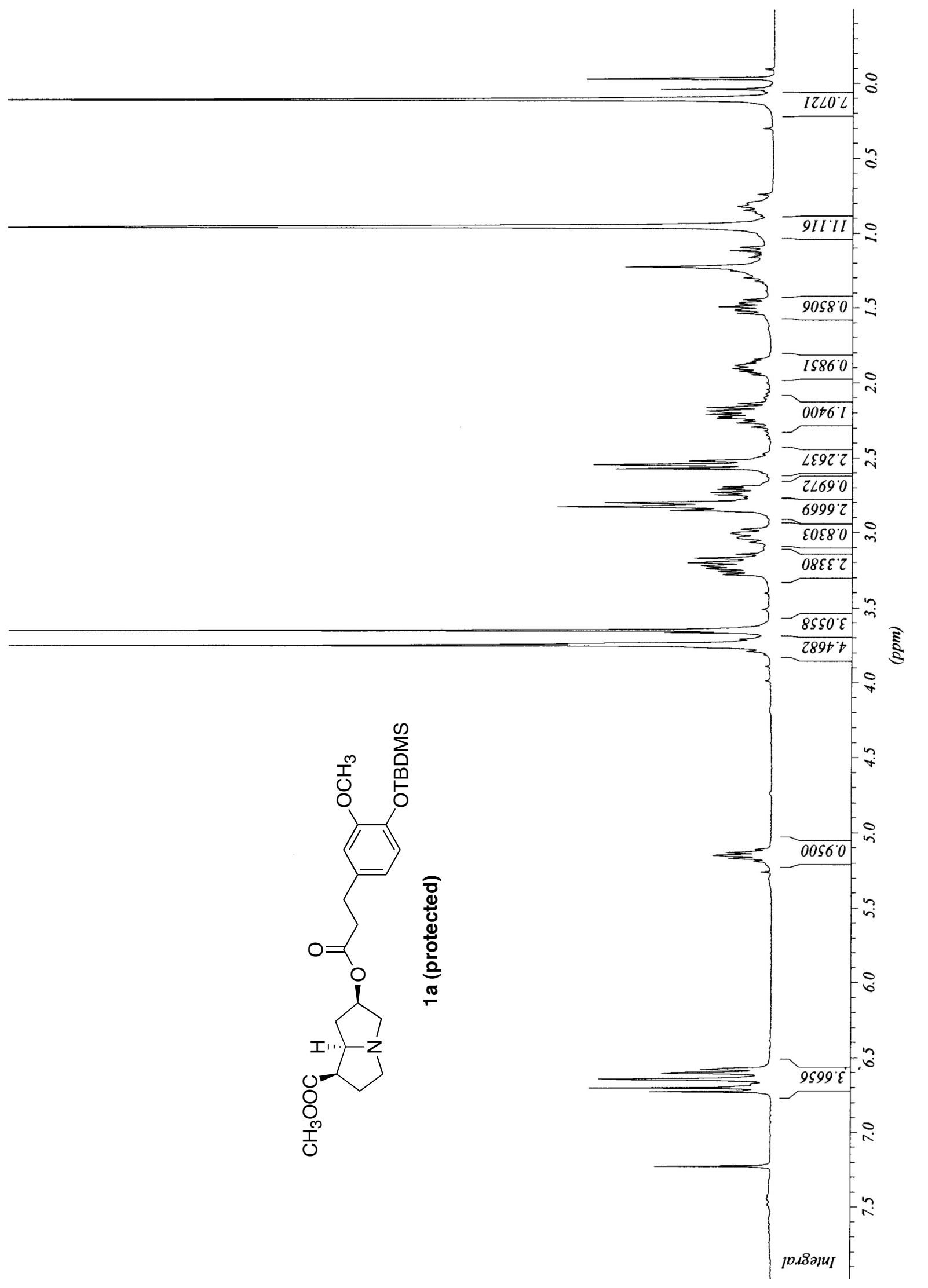




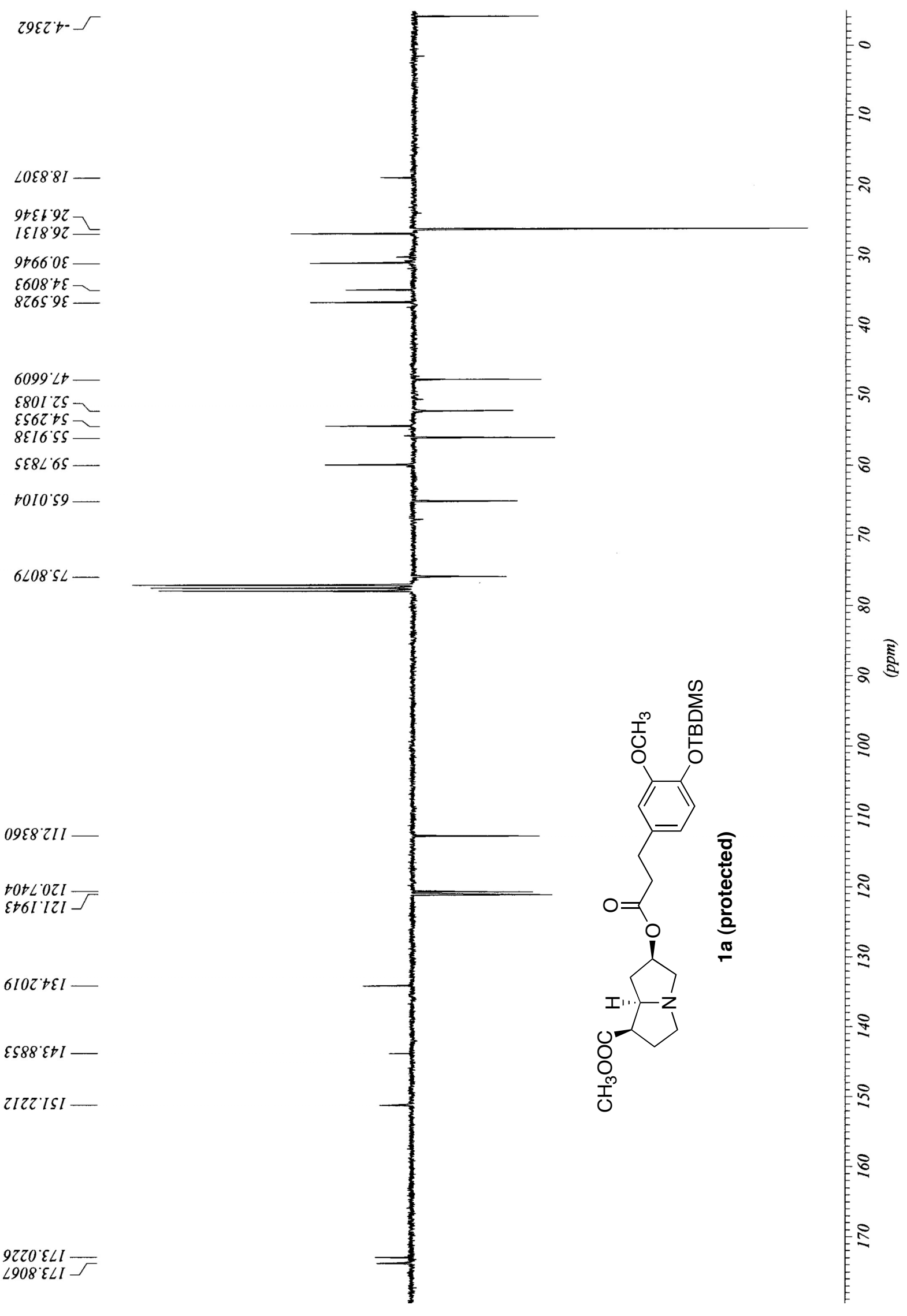




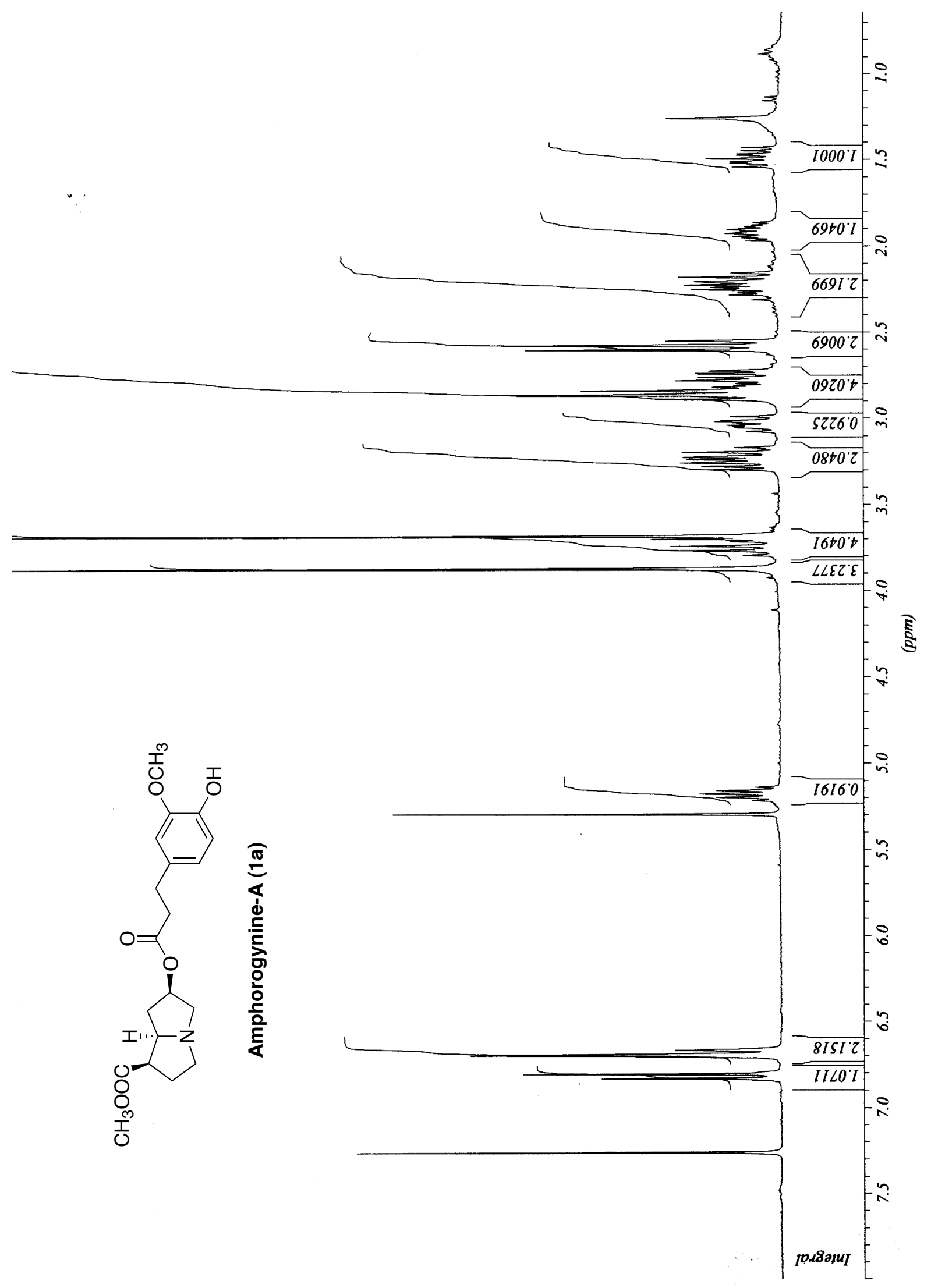



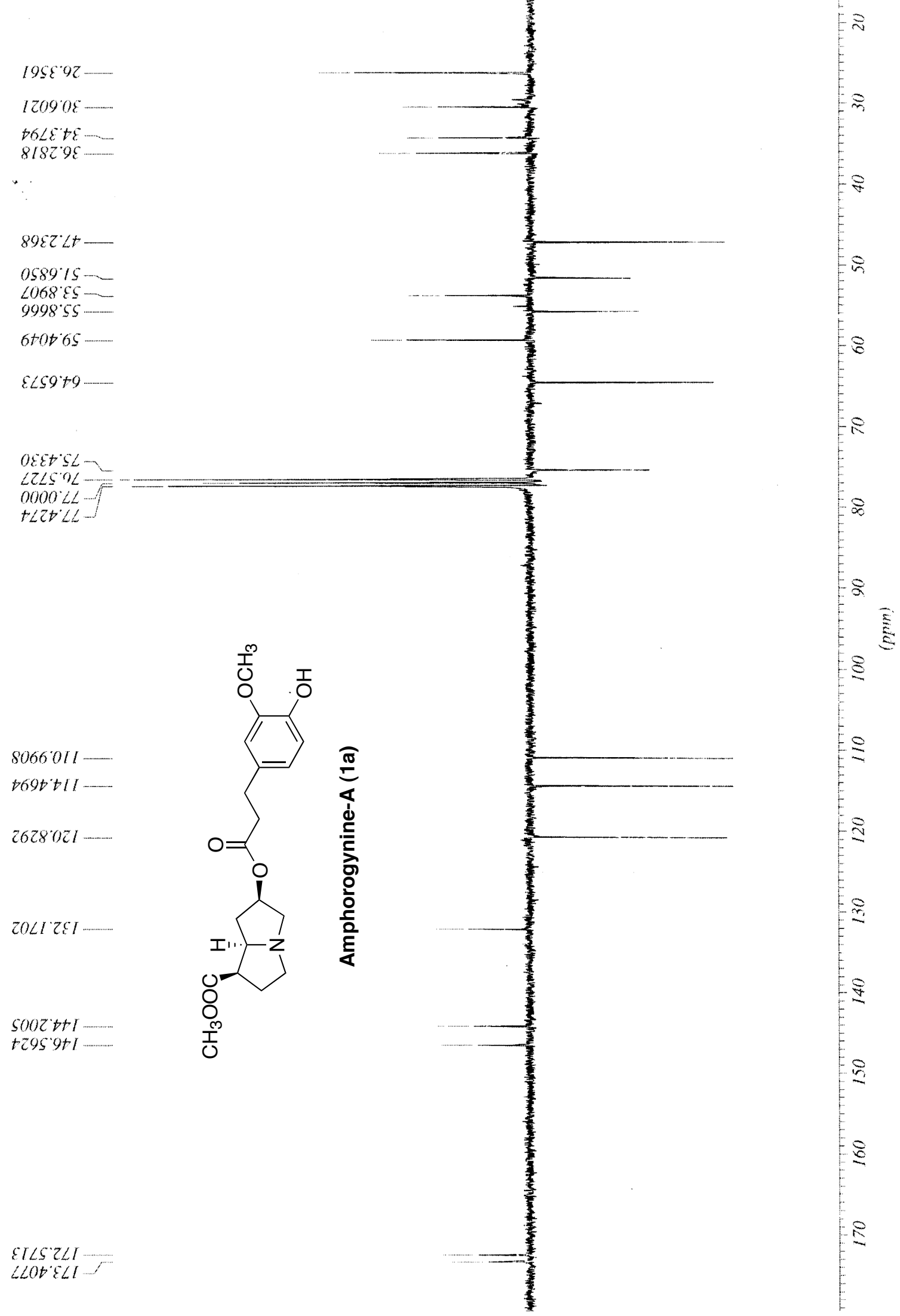


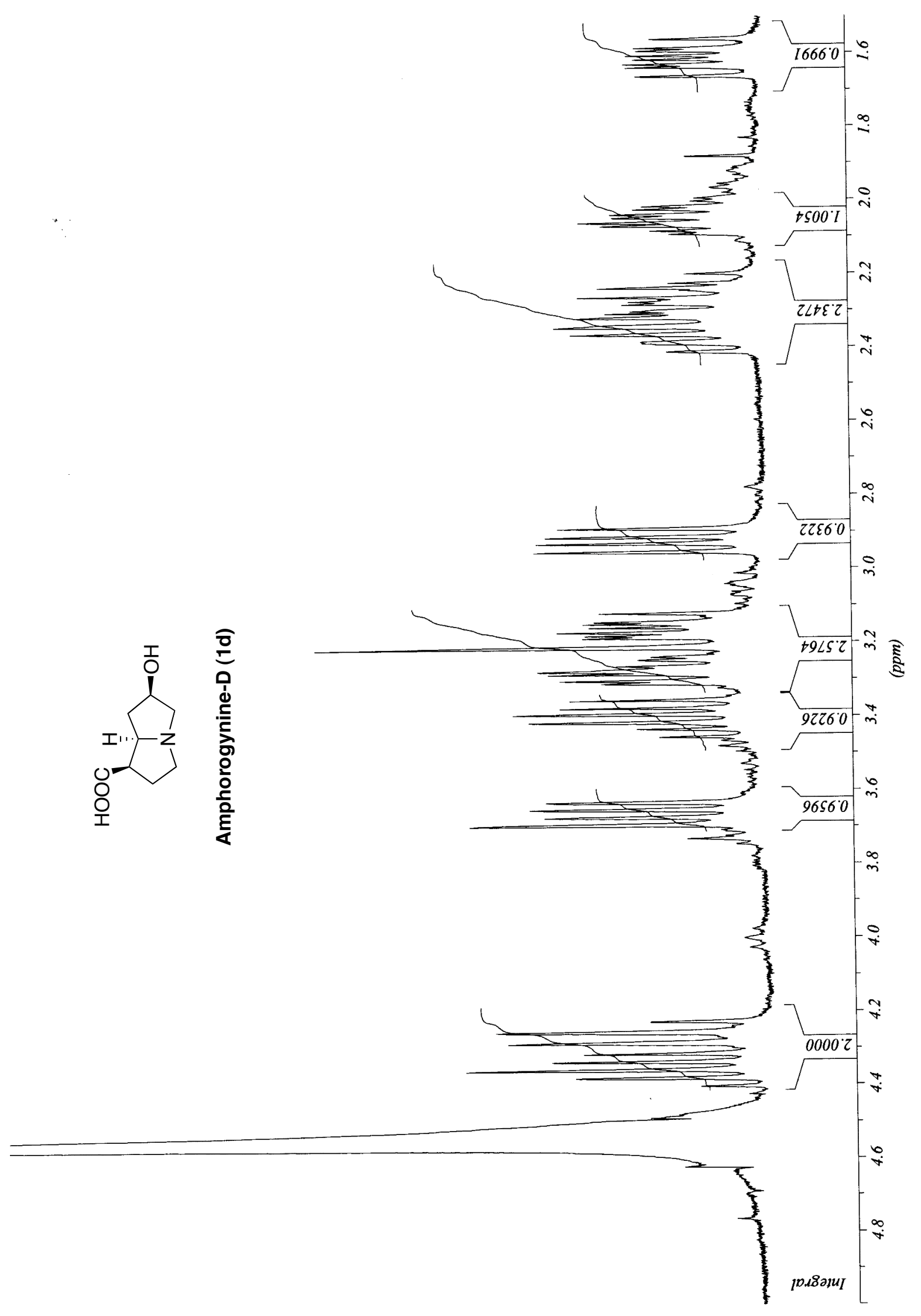




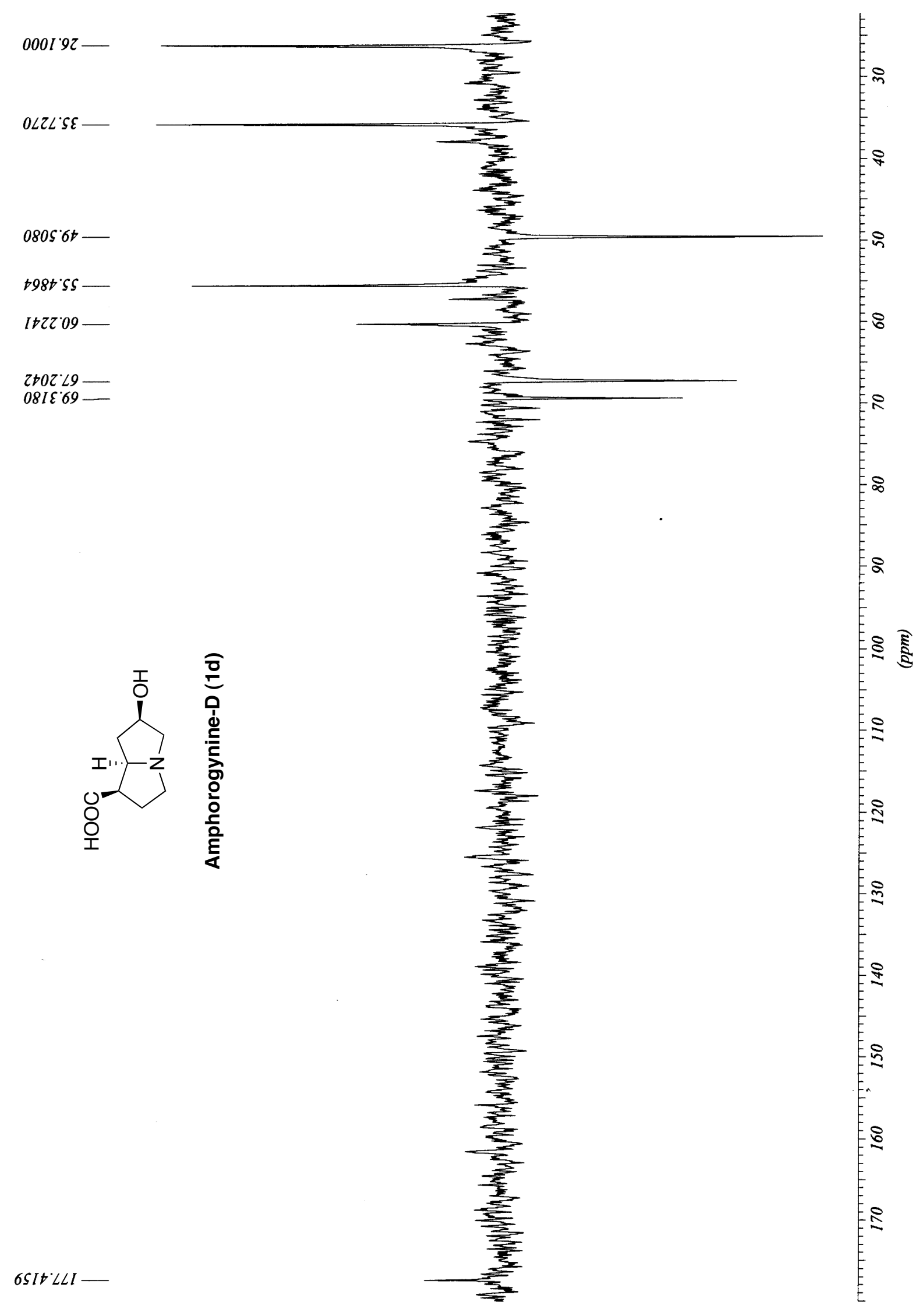




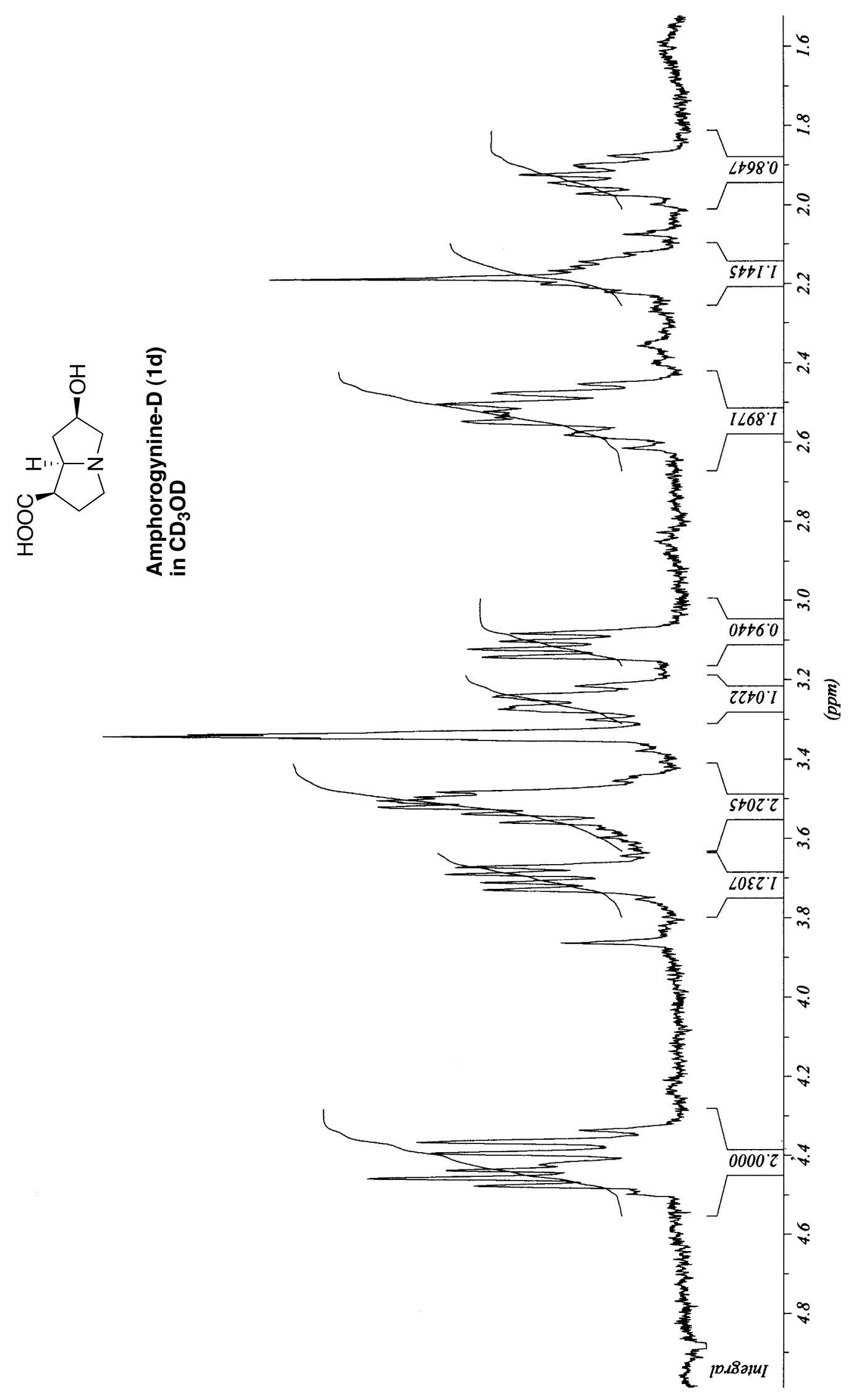




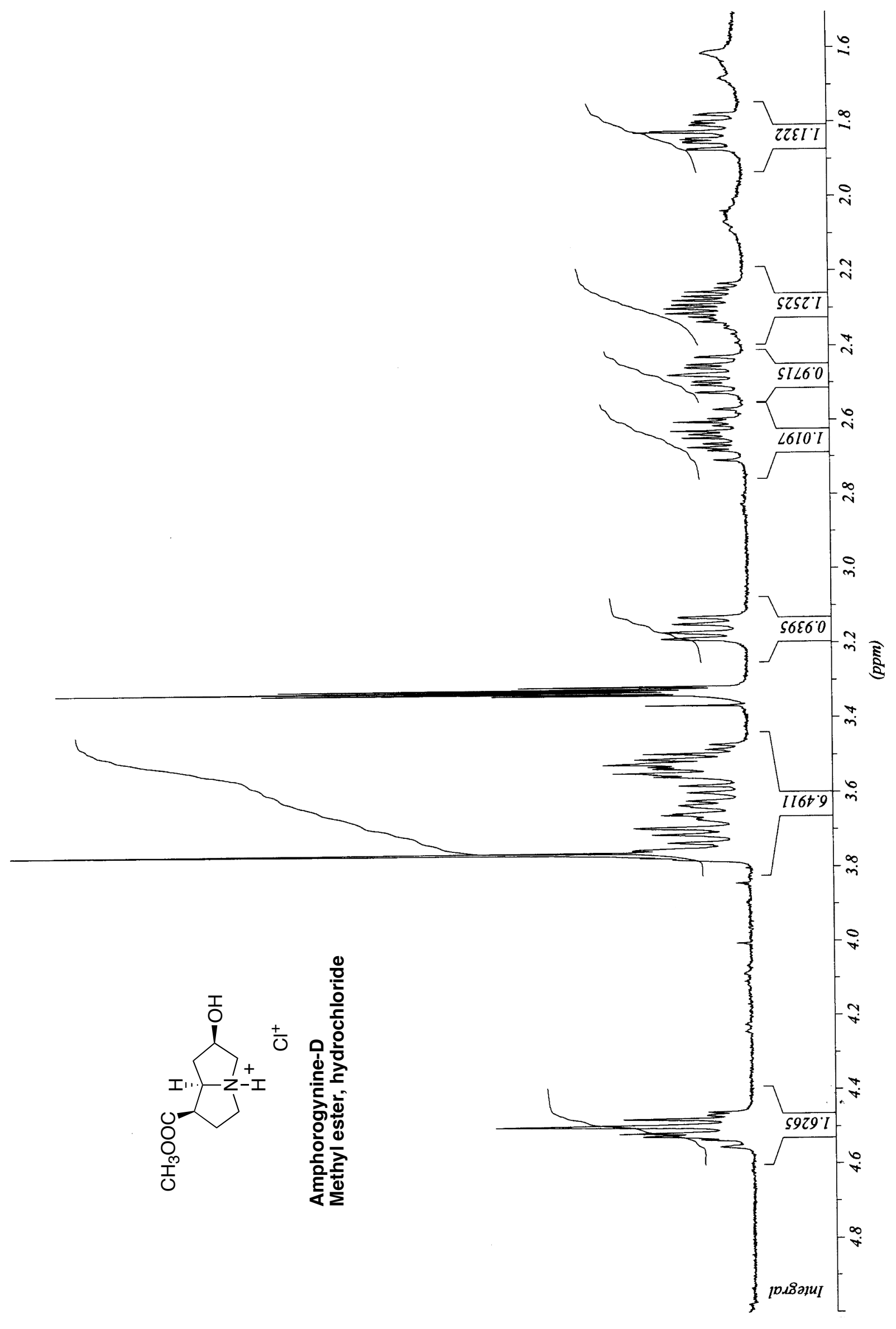




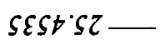

$\angle 8+t^{\circ} S \mathcal{-}$
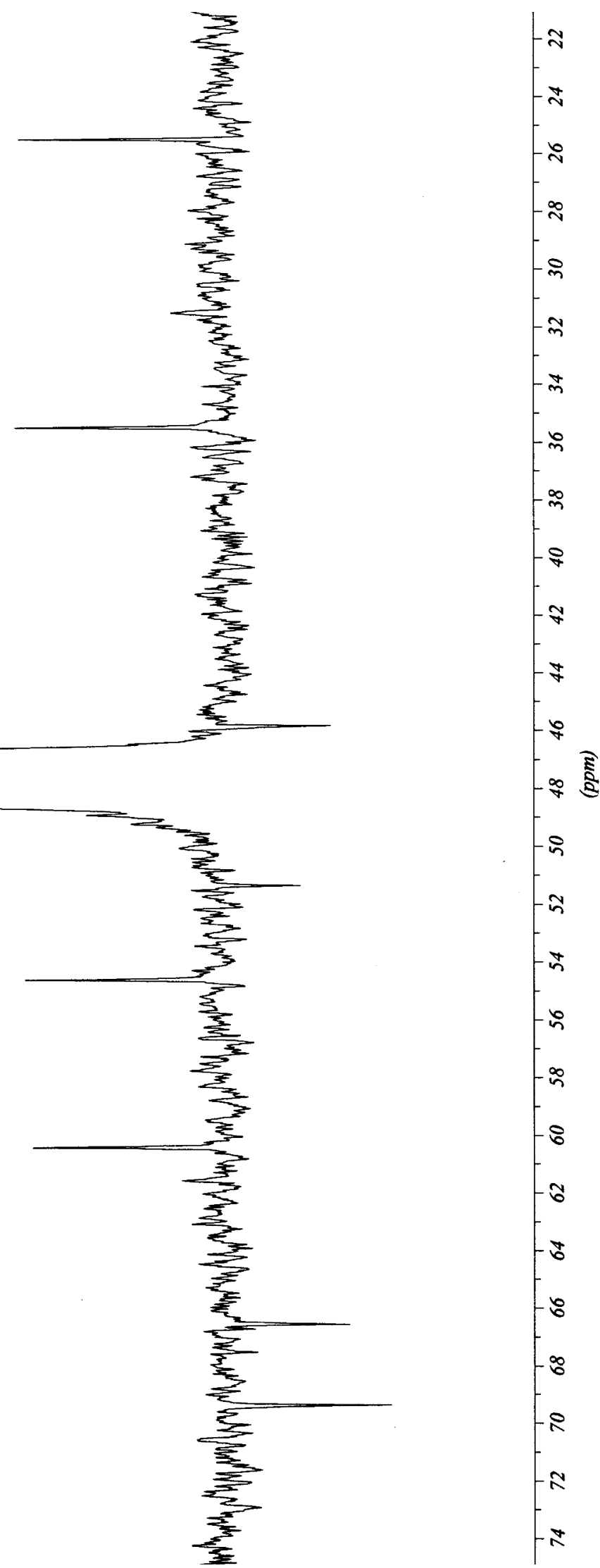

IOtE IS

$0009 \% 5$

000009

$9+\varepsilon 5.99$

$68 S \mathcal{6} 69$
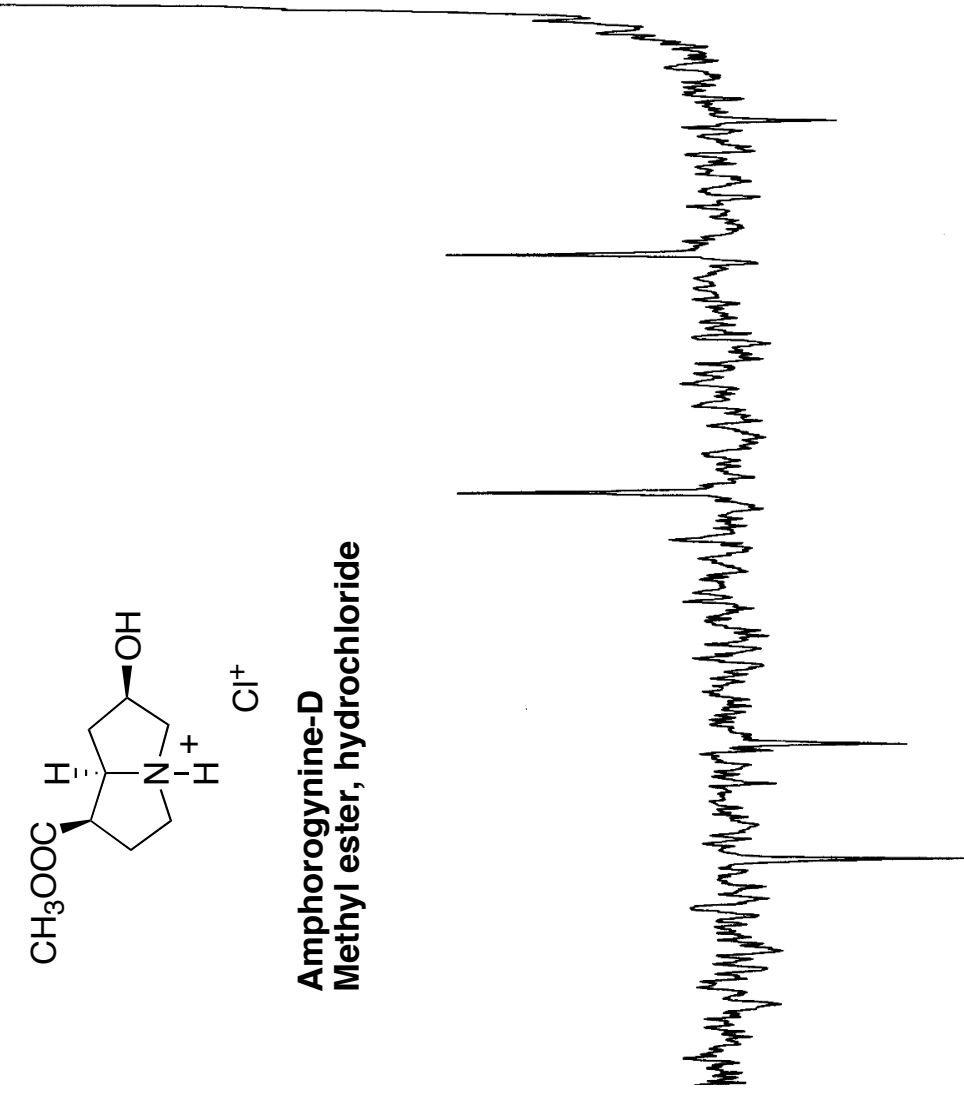


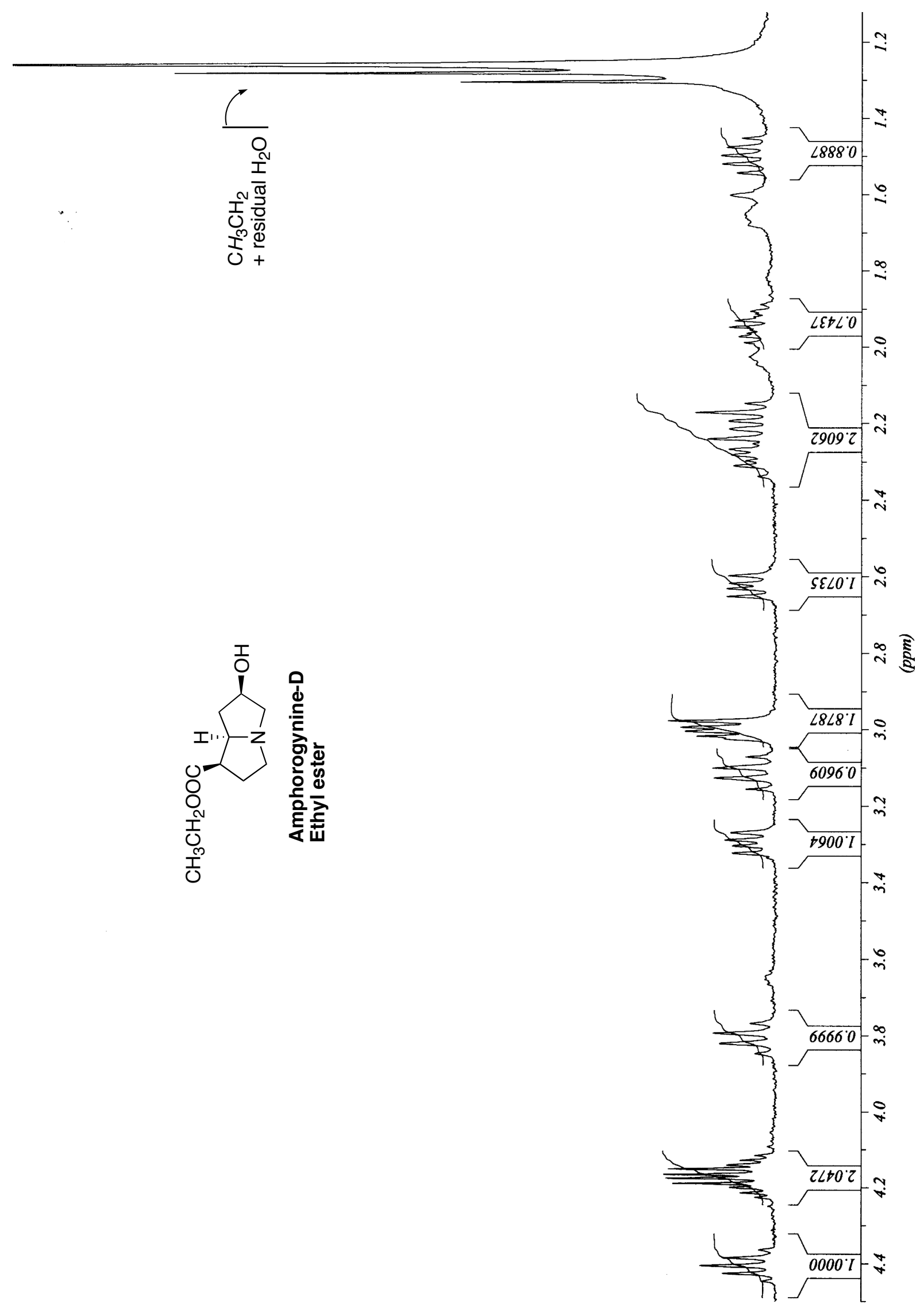


$\sigma \subseteq 6 \tau^{\circ} \mapsto I$

$\$ 8 \varsigma \varepsilon \angle Z-$

$0 S I L 6 Z$

$\phi \tau \varepsilon \varsigma^{\prime}<\varepsilon-$

$\angle S 90^{\circ}+5$

$18 \angle 409$

I698: 29

$8 \angle S \angle t 9$

$+919.2 L$

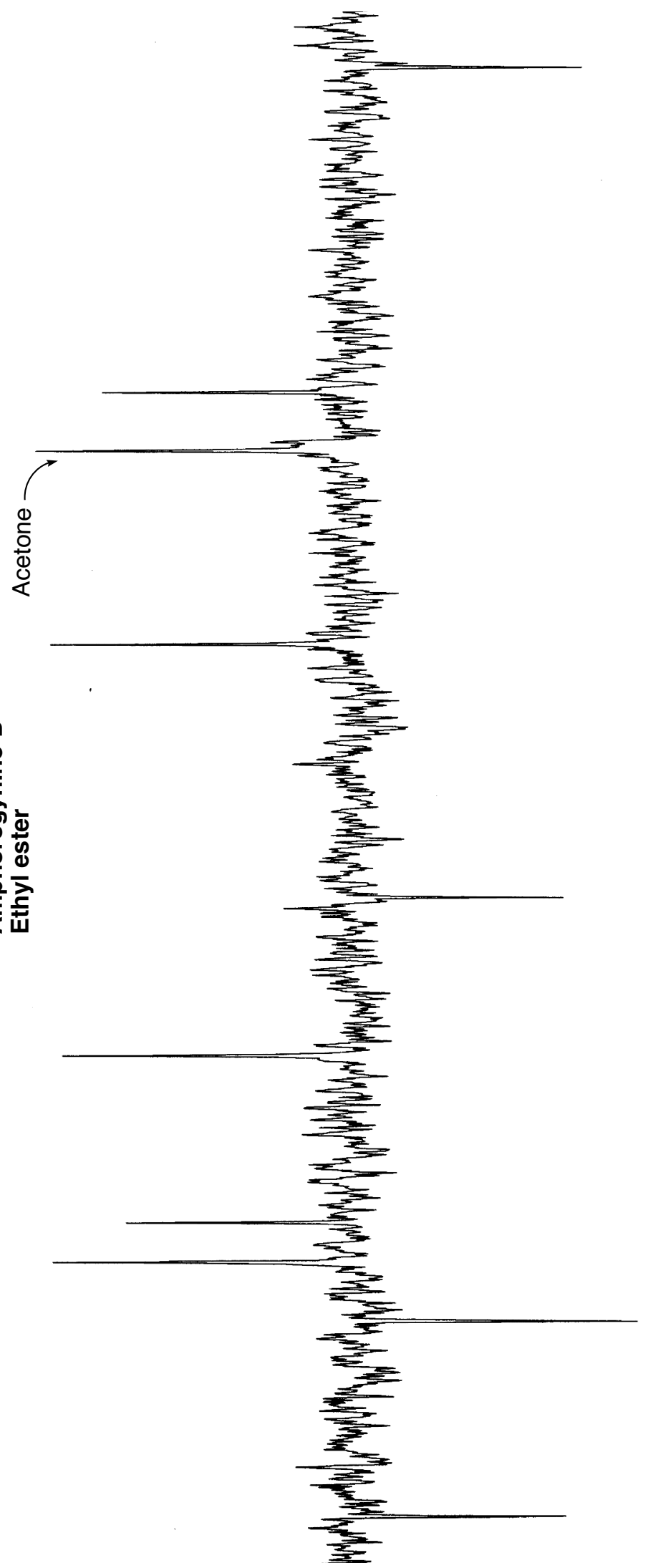




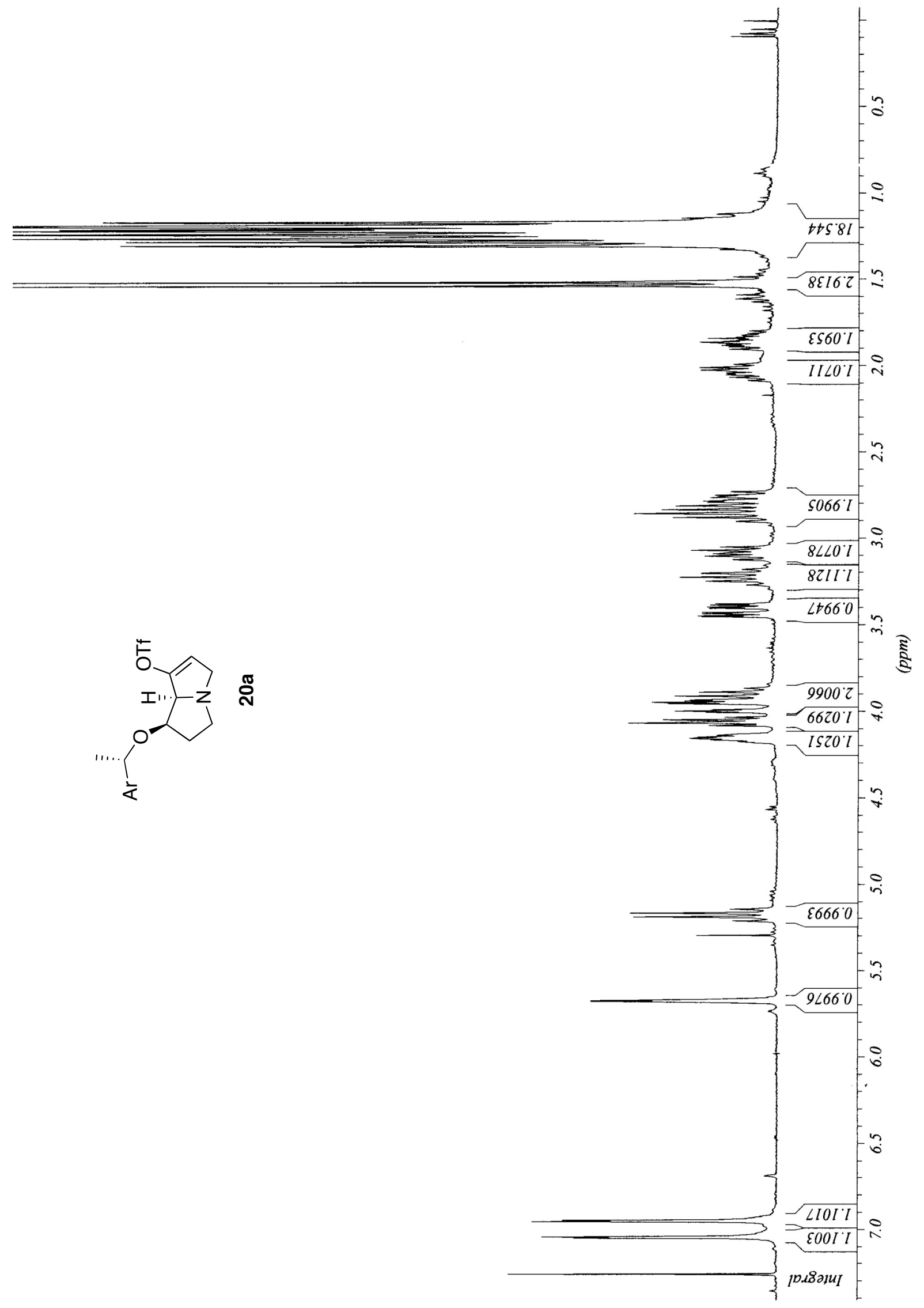


$Z \angle I I^{\circ} \mathcal{E} Z$

$8 S 10^{\circ}+2$

$6<+0 \div z$

$6 \varepsilon I \varepsilon+\tau$

$\angle 99 I^{\circ} \mathrm{SZ} \longrightarrow$

$6292.52 \perp$

$2011^{\circ} 82$

$\varepsilon+27.6 z$

$\angle \mathcal{E O I} Z \mathcal{E}$

I860"

$09 I I+5$

1628.65

$68+9 \% \mathrm{~L}$

$\tau \varepsilon+0^{\circ} Z L$

28EL $9 \angle$

$0091^{\circ} \angle L-5$

$\angle 90 \mathcal{E}^{\circ} \angle L-$

$+985^{\circ} \mathrm{LL}-$

st62'ZII

SSOI + II

LttS.9II

$966 \angle 0 Z I$

$0058^{\circ} 0 z I$

$008 \mathrm{CZl}^{\mathrm{E}}$

$+\oplus 50^{\circ} \mathrm{SZI}-$

$99 \angle \mathcal{E} Z \varepsilon I$

$6 \angle 9 S+D I$

$t s+9.5+5$

$8+85^{\circ} \mathrm{LH}-$

$91006 \mathrm{t}$

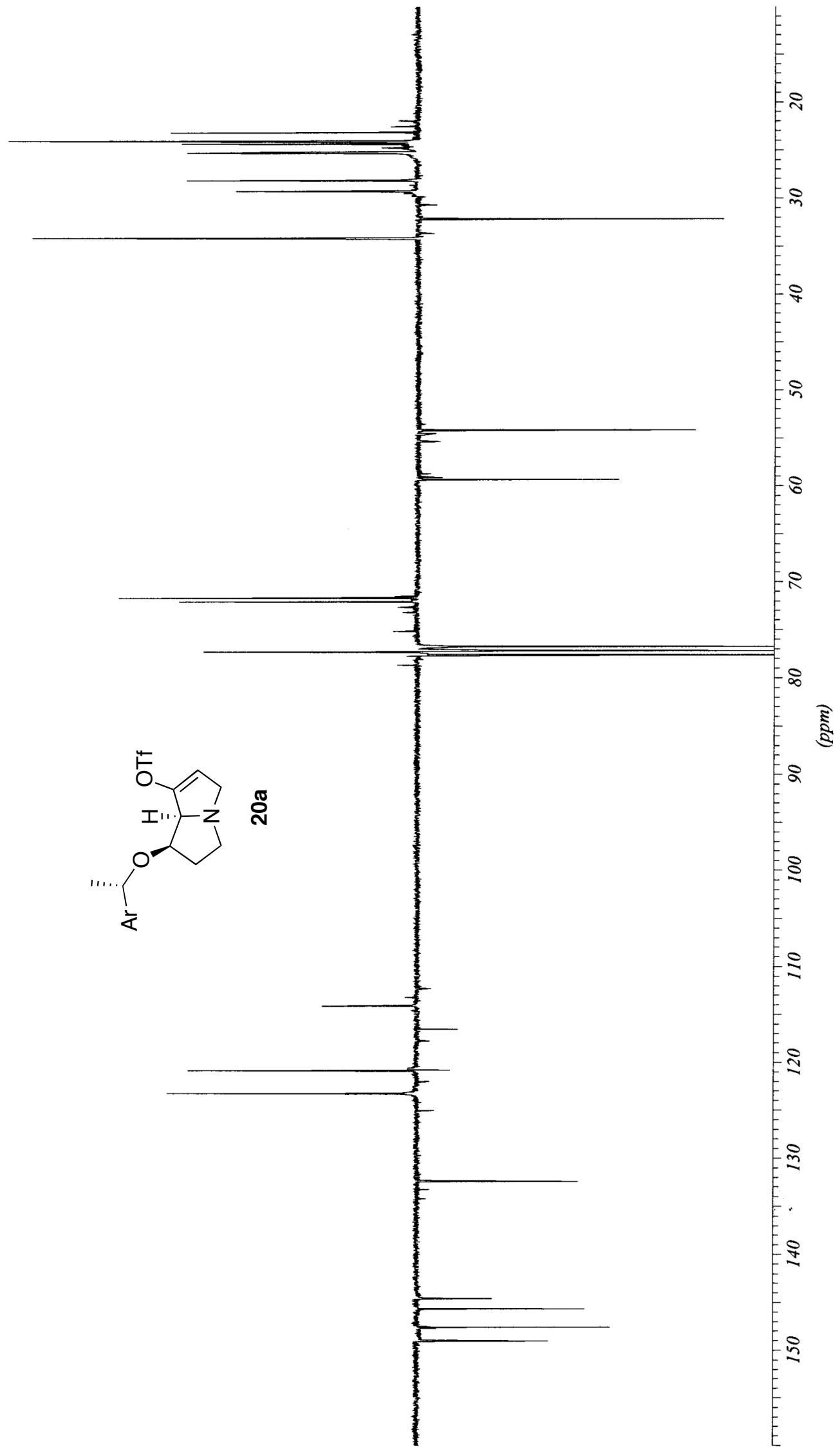




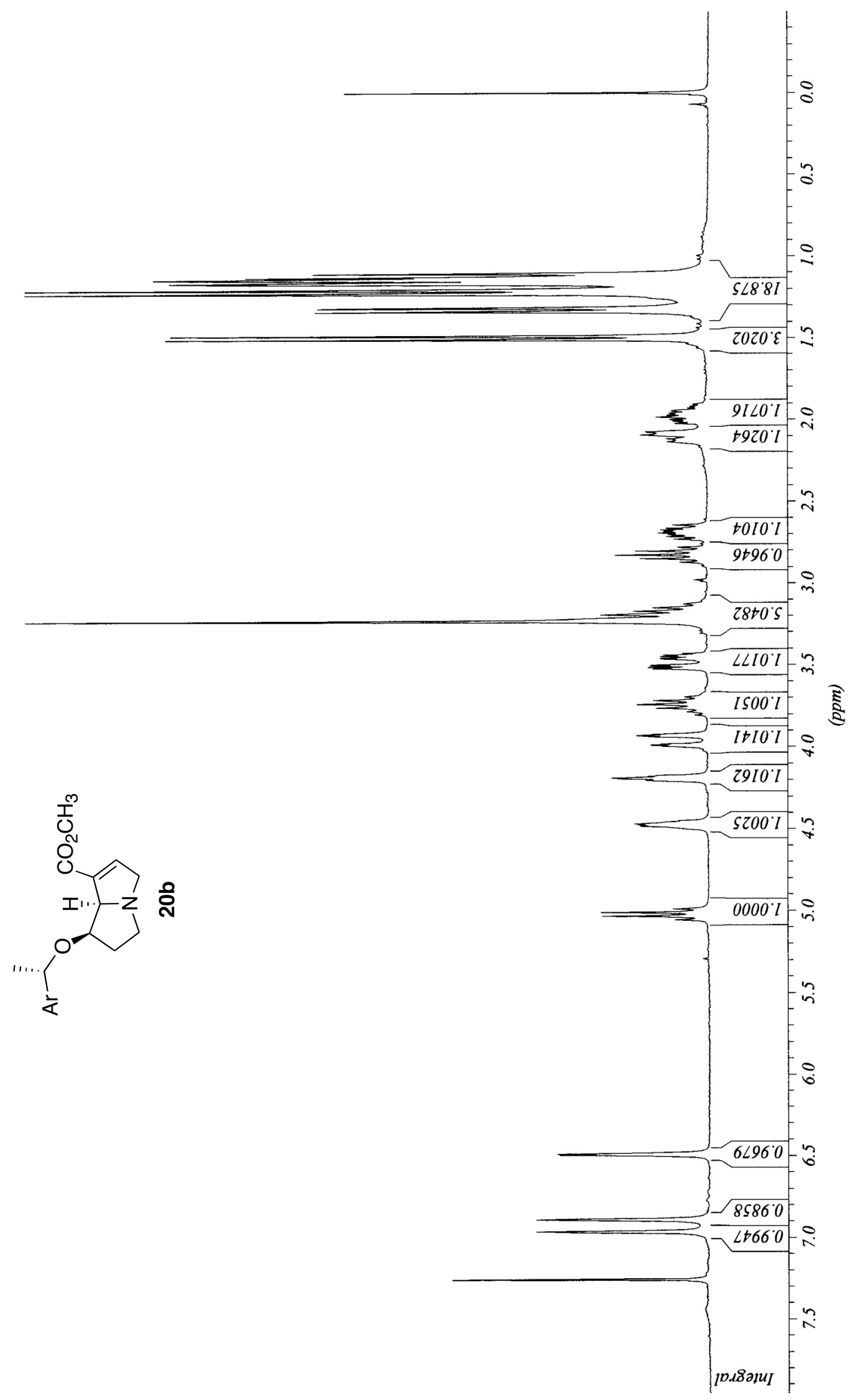




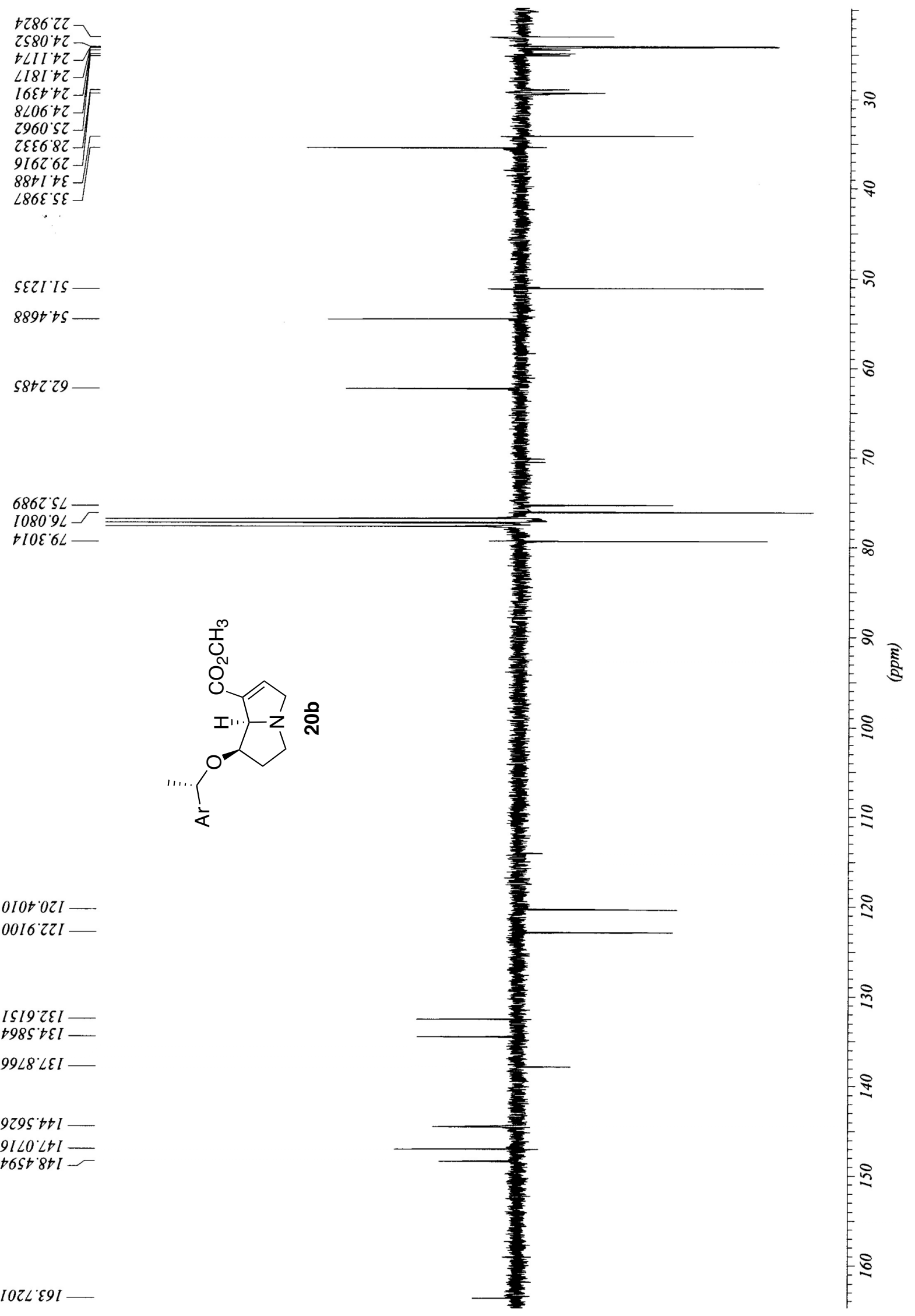




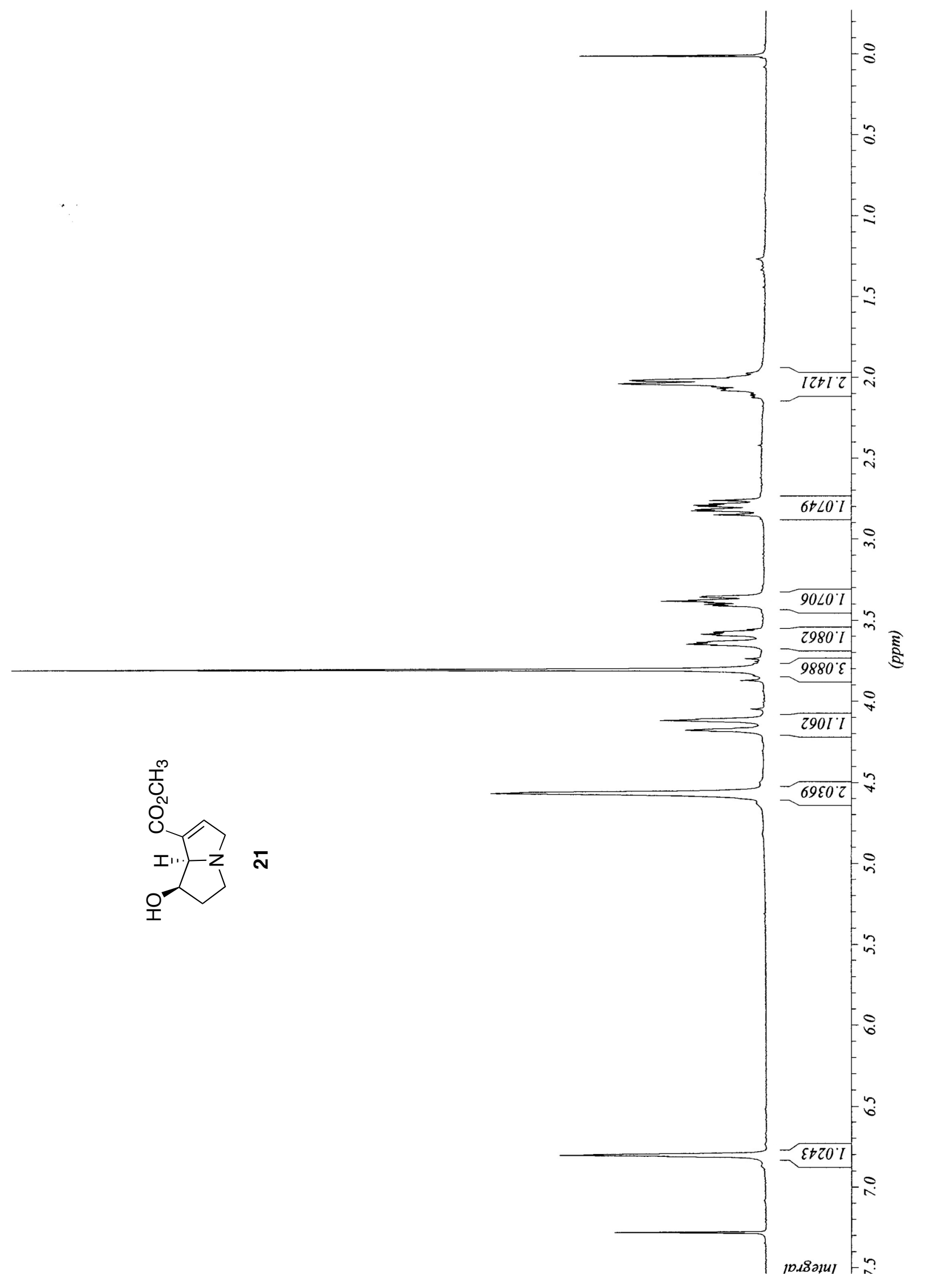




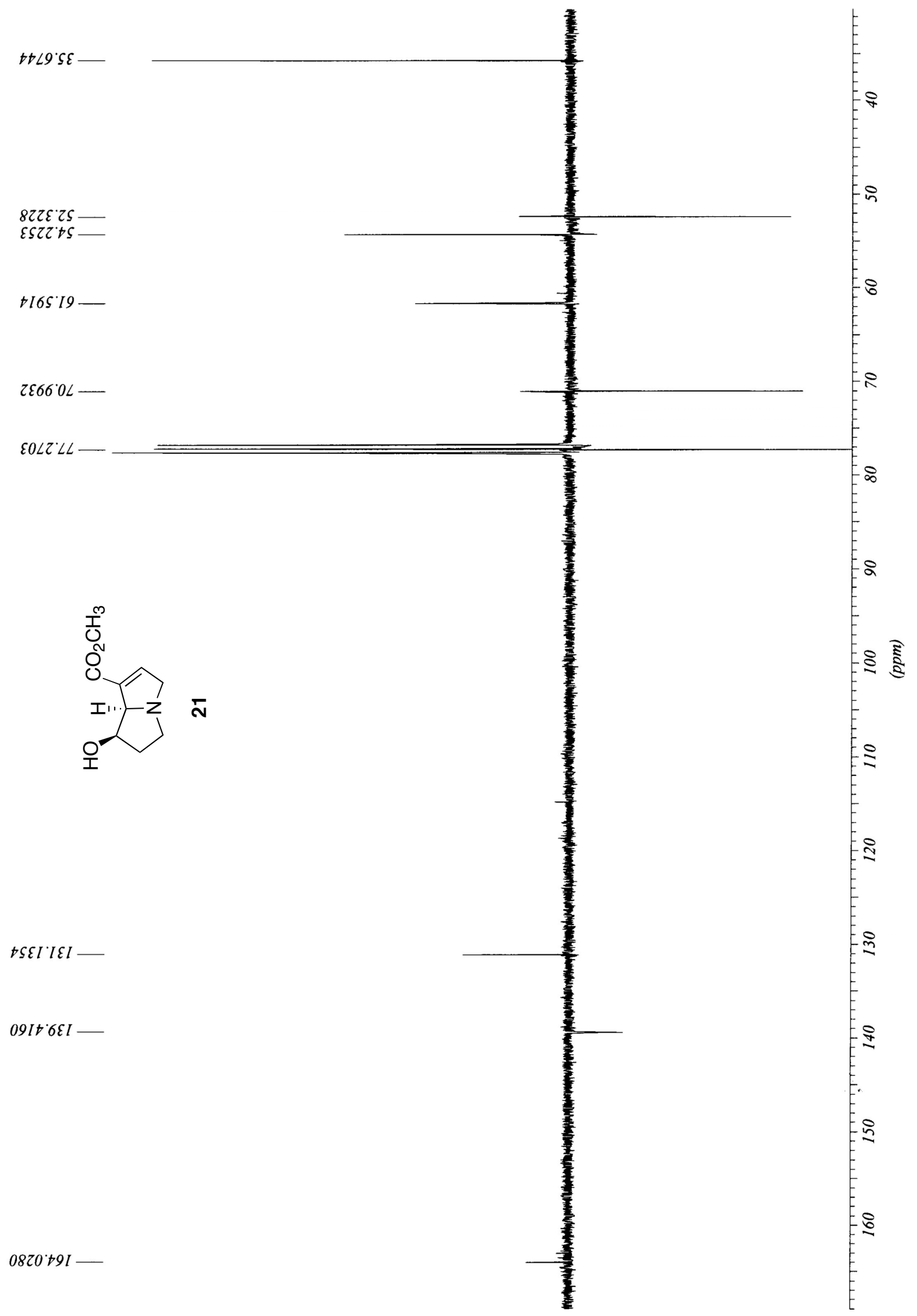




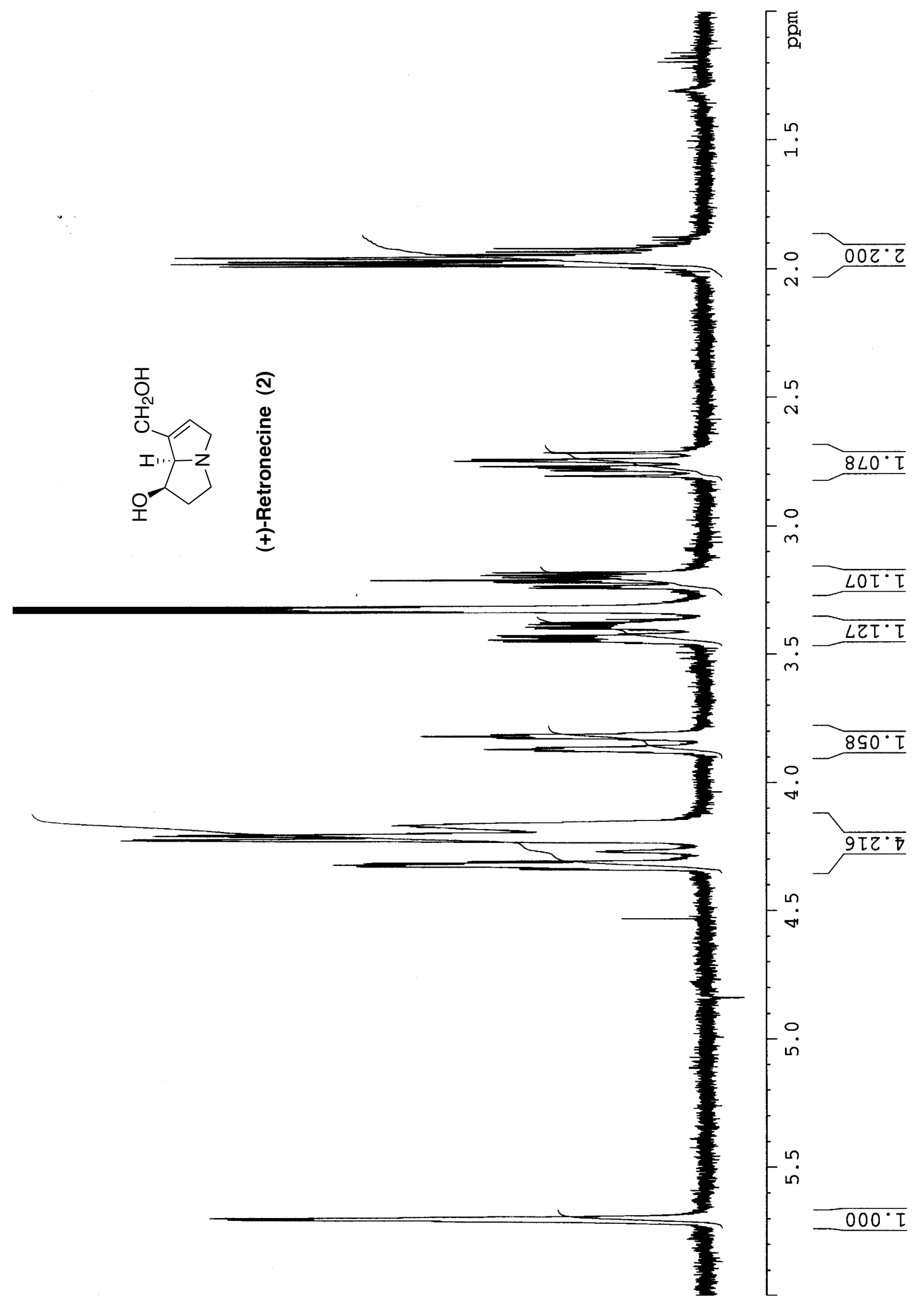




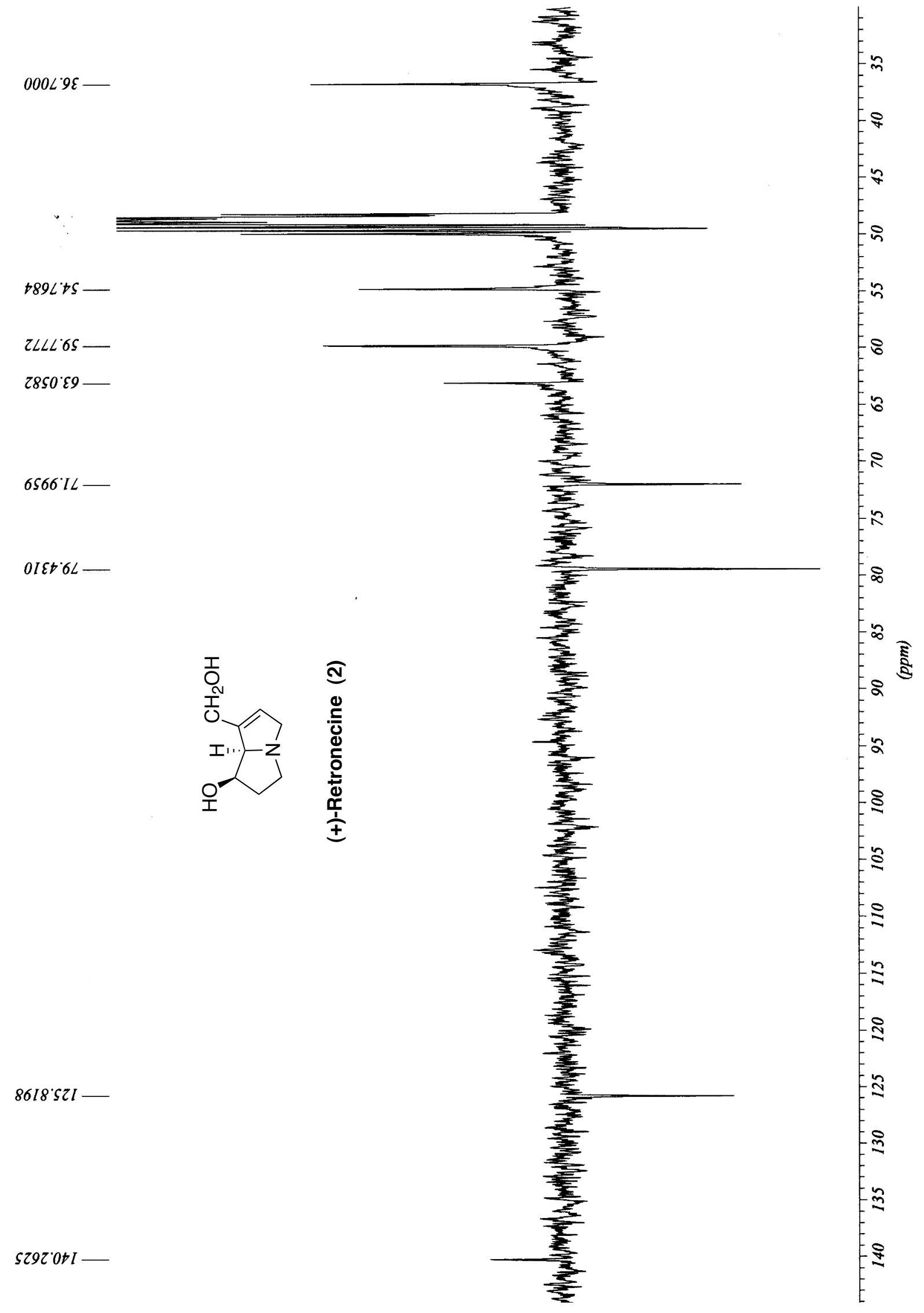

